\title{
Hedgehog signaling modulates cigarette-induced COPD development
}

\author{
FANG YING LU ${ }^{1,2^{*}}$, RONG CHEN $^{1,2^{*}}$, MIN ZHOU $^{1,2}$ and YI GUO $^{1,2}$ \\ ${ }^{1}$ Department of Respiratory and Critical Care Medicine, Shanghai Rui Jin Hospital, School of Medicine; \\ ${ }^{2}$ Institute of Respiratory Diseases, School of Medicine, Shanghai Jiao Tong University, Shanghai 200025, P.R. China
}

Received July 19, 2020; Accepted February 1, 2021

DOI: $10.3892 /$ etm.2021.10161

\begin{abstract}
Hedgehog (Hh) signaling is involved in early embryogenesis and maintains quiescence in the adult lungs. The interruption of Hh signaling may lead to the development of chronic obstructive pulmonary disease (COPD). The current study aimed to assess whether the Hh pathway affects cigarette-induced emphysema and airway inflammation by regulating inflammatory cytokines. C57BL/6J mice were randomized into control, cigarette smoke (CS) or CS + cyclopamine (CSC) groups. Control mice were exposed to normal room air, CS mice were exposed to tobacco smoke and CSC mice were exposed to $\mathrm{CS}$ and received cyclopamine treatment. Histopathological examination of lung tissues was performed, and the expression of sonic hedgehog (HH), glioma-associated oncogene homolog 1 (Gli1), hedgehog-interacting protein (HIP) and several inflammatory mediators (intracellular adhesion molecule-1, IL-6, IL-8 and TNF- $\alpha$ ) were compared using reverse transcription-quantitative PCR and western blotting. The emphysema of lung tissues by histopathological examination demonstrated partial amelioration in the CSC group compared with that in the CS group. Additionally, expression levels of SHH, Gli1 and inflammatory mediators were significantly higher in the CS group compared with the control group but were significantly decreased in the CSC group. The
\end{abstract}

Correspondence to: Dr Yi Guo, Department of Respiratory and Critical Care Medicine, Shanghai Rui Jin Hospital, School of Medicine, Shanghai Jiao Tong University, Building 36, 197 Rui Jin Er Road, Shanghai 200025, P.R. China

E-mail: guoyi621@qq.com

${ }^{*}$ Contributed equally

Abbreviations: COPD, chronic obstructive pulmonary disease; CS, cigarette smoke; DHH, desert hedgehog; Gli1, Glioma-associated oncogene homolog; Hh, hedgehog; HIP, hedgehog-interacting protein; ICAM-1, intercellular cell adhesion molecule-1; IHH, Indian hedgehog; Ptc, patched; SHH, sonic hedgehog; Smo, smoothened

Key words: hedgehog signaling, cigarette smoke, chronic obstructive pulmonary disease development, inflammatory mediators, emphysema expression of HIP was decreased in the CS group, but significantly increased in the CSC group. Hh signaling may serve an important role in cigarette-induced emphysema and airway inflammation by regulating inflammatory cytokines in animal models. Therefore, diminishing the activation of the Hh signal may serve as a novel therapeutic strategy for patients suffering from smoking-related COPD.

\section{Introduction}

Chronic obstructive pulmonary disease (COPD) involves persistent airway inflammation and emphysema; it is primarily caused by cigarette smoke (CS) and genetic predisposition (1). It is characterized by tissue remodeling, emphysematous alveolar destruction, enlarged air spaces and chronic airway inflammation. The two major pathological changes of COPD include emphysema and airway inflammation. Previous research has focused on the underlying molecular mechanisms that lead to COPD progression, and some of the pathological processes are mediated by the abnormal activation of certain signaling pathways (2); however, the mechanisms that initiate the disease are not fully understood.

Hedgehog (Hh) signaling is one of the pathways involved in early embryogenesis, serving an important role in cell growth, differentiation, tissue patterning and vascularization (3). Sonic hedgehog (SHH), desert hedgehog (DHH) and Indian hedgehog (IHH) are three ligands that initiate the canonical Hh signaling pathway. SHH is a predominant ligand in the adult lung, which is expressed by the proximal airway and distal alveolar epithelium. The ligand binding of $\mathrm{Hh}$ to its repressive receptor, patched (Ptc; a twelve-transmembrane protein), causes the suppression of signal transduction, relieving smoothened (Smo; a seven-transmembrane protein) from Ptc and subsequently activating the signaling cascade (3). This leads to the translocation of certain transcription factors, including glioma-associated oncogene homolog (Gli) 1, Gli2 and Gli3, to the nucleus, therefore involving many downstream target genes that initiate transcription (4). In particular, Gli1 acts as the most important molecule in the human body. Cyclopamine is a steroid alkaloid that inhibits Hh signaling by directly binding to Smo (4).

Previous studies (3-5) have demonstrated that Hh signaling maintains mesenchymal quiescence in the adult lung; however, repeated exposure to $\mathrm{CS}$ causes the aberrant activation of $\mathrm{Hh}$ 
signaling, leading to its overexpression in bronchial epithelial cells. Additionally, $\mathrm{Hh}$ signaling is capable of promoting the airway inflammatory process, inducing abnormal alveolar and bronchial epithelial cell apoptosis, which may facilitate COPD initiation and progression (5). The interruption of Hh signaling at earlier time points (embryon) results in enlarged alveolar airspaces. Signaling is also dysregulated in diseases such as COPD (6). Hedgehog-interacting protein (HIP) is a feedback inhibitor of Hh. It competitively binds to three Hh ligands and has an affinity equal to that of Ptc, thus leading to the repression of the Hh signaling pathway (7). HIP has a protective role in COPD pathogenesis, and according to studies by Lao et al $(7,8), 32 \%$ of patient COPD lung tissues exhibited a reduced expression of the HIP gene compared with smokers without airflow limitation.

Our previous study indicated that $\mathrm{Hh}$ served a role in cigarette-induced airway inflammation through the regulation of certain inflammatory cytokines in an A549 cell model (9); however, the role of $\mathrm{Hh}$ in emphysema was not elucidated. The current study therefore assessed the effect of the Hh signaling pathway on COPD progression, which is a possible molecular mechanism of cigarette-induced emphysema and airway inflammation. This was evaluated by measuring the expression of SHH, Gli1, HIP and various airway inflammatory mediators using an animal model.

\section{Materials and methods}

Animals. All animal experiments were approved by the Institutional Animal Care and Use Committee of Shanghai Rui Jin Hospital, Shanghai Jiao Tong University (Shanghai, China; approval no. 105). A total of 30 12-week-old C57BL/6J male mice $(31.7 \pm 4.2 \mathrm{~g})$ were purchased from Shanghai SLAC Laboratory Animal Co., Ltd. These mice were housed in a pathogen-free, temperature-controlled environment with $24^{\circ} \mathrm{C}$ and $40 \%$ humidity under a $12: 12 \mathrm{~h}$ light-dark cycle, with wood chip bedding, food and water were available ad libitum throughout experiments. After 2 weeks of adaptation, mice were randomly divided into three groups ( $\mathrm{n}=10 \mathrm{mice} / \mathrm{group}$ ): Control, CS and CS + cyclopamine (CSC). In the control group, $\mathrm{C} 57 \mathrm{BL} / 6 \mathrm{~J}$ mice were exposed to normal room air. The mice in CS group were exposed to the tobacco smoke produced from five cigarettes, four times per day for $30 \mathrm{~min}$, for a total of 5 days per week for 24 weeks. Mice were subsequently anesthetized with an intraperitoneal injection of ketamine $(60 \mathrm{mg} / \mathrm{kg})$ and xylazine $(8 \mathrm{mg} / \mathrm{kg})(10)$, after which lipopolysaccharide (LPS; 0.9 mg/kg body weight; Sigma-Aldrich; Merck KGaA) was administrated intratracheally each day in the last week, as previously described (11). One week following LPS injections, mice in CSC group were administered intraperitoneal injections of $50 \mathrm{mg} / \mathrm{kg}$ cyclopamine (12).

Histopathological examination. Mice were euthanized by an intraperitoneal injection of sodium pentobarbital $(100 \mathrm{mg} / \mathrm{kg})$, and lung tissues were carefully removed. Hematoxylin and eosin (H\&E) staining was performed to observe the pathological changes of the lung. Lung tissues were obtained from the mice of each group and fixed using $4 \%$ formaldehyde in PBS in $4^{\circ} \mathrm{C}$ overnight, after which tissues were dehydrated using a Leica TP 1020 Rapid Tissue Processor (Leica, Inc.).
Samples were then embedded in paraffin and serial sections (4 $\mu \mathrm{m}$ thick) were stained H\&E staining (Servicebio, Inc.) in room temperature. All experiments were performed according to the manufacturers' instructions. To observe the morphology of lung tissues, slides were evaluated under a Leica photograph light microscope (Leica Microsystems $\mathrm{GmbH}$; magnification, $\mathrm{x} 200$ ).

Reverse transcription-quantitative PCR (RT-qPCR) analysis of SHH, Glil, HIP and other inflammatory mediators. A total of $\sim 3 \mathrm{~g}$ tissues of each samples were used for PCR analysis. Tissues were ground into fragments in PBS. Total RNA was extracted using TRIzol ${ }^{\circledR}$ reagent (Ambion; Thermo Fisher Scientific, Inc.) from the dissolved lung tissues. cDNA was synthesized using Moloney Murine Leukemia Virus Reverse (M-MLV) reverse transcriptase (Takara Biotechnology Co., Ltd.). PCR was performed using ChamQ SYBR Color qPCR Master Mix (Vazyme Biotech Co., Ltd.). Briefly, $2.5 \mu \mathrm{l}$ of $20 \mu \mathrm{M}$ oligo (dT) primer stock was added to the RNA sample, after which RNase-free $\mathrm{H}_{2} \mathrm{O}$ was also added to a final volume of $11.5 \mu \mathrm{l}$. The mixture was incubated at $70^{\circ} \mathrm{C}$ for $3 \mathrm{~min}$. Next, $4 \mu 1$ 5X Reverse Transcription Buffer, $2 \mu \mathrm{l}$ dNTP Mix, $2 \mu 1$ $100 \mathrm{mM}$ DTT and $0.5 \mu \mathrm{l}$ M-MLV Reverse Transcriptase was added. Samples were then incubated at $42^{\circ} \mathrm{C}$ for $60 \mathrm{~min}$ and the reaction was terminated by incubation at $70^{\circ} \mathrm{C}$ for $15 \mathrm{~min}$. An ABI 7900 Real-Time PCR System (Thermo Fisher Scientific, Inc.) was used to monitor amplification reactions in real-time. The primers used for PCR were as follows: SHH forward, 5'-AAGCGCGCTCTTTGCCA-'3 and reverse, 5'-TTGATG AGAATGGTGCCGTG-3'; Gli1 forward, 5'-TTGCAGCCA GGAGTTCGATT-3' and reverse, 5'-TGTGCACCACCAGCA TGTAT-3'; HIP forward, 5'-GAAGAACGCAGAGGTGAC CA-3' and reverse, 5'-CCTGTTGCTCTTGAGTCCGT-3'; intracellular adhesion molecule (ICAM-1) forward, 5'-CTG GGCTTGGAGACTCAGTG-3' and reverse, 5'-CCACAC TCTCCGGAAACGAA-3'; IL-6 forward, 5'-CACTTCACA AGTCGGAGGCT-3' and reverse, 5'-CTGCAAGTGCATCAT CGTTGT-3'; IL-8 forward, 5'-CTAGGCATCTTCGTCCGT CC-3' and reverse, 5'-TTCACCCATGGAGCATCAGG-3'; TNF- $\alpha$ forward, 5'-AGGCACTCCCCCAAAAGATG-3' and reverse, 5'-TGAGGGTCTGGGCCATAGAA-3'; GAPDH forward, 5'-CTCCTCCTGGCCTCGCTGT-3' and reverse, 5'-GCTGTCACCTTCACCGTTCC-3'. Data were evaluated using the $2^{-\triangle \triangle C q}$ method (13).

Western blotting of SHH, Gli1, HIP and inflammatory mediators. RIPA buffer (Sigma-Aldrich; Merck KgaA) containing protease inhibitors was used to lyse and extract protein from lung tissue ( $3 \mathrm{~g}$ tissues of each samples). A Bradford assay was subsequently performed to detect the total protein concentration of lung tissue. Equal amounts of protein were separated by TGX Stain-Free FastCast Acrylamide Kit $12 \%$ gel (Bio-Rad, Inc.), and subsequently transferred onto a $0.45 \mu \mathrm{m}$ PVDF membrane (GE Healthcare, Inc.). Membranes were then incubated with primary antibodies (Table SI) overnight at $4^{\circ} \mathrm{C}$, after which membranes were probed with secondary antibodies for $1 \mathrm{~h}$ at room temperature as described previously (Table SI) (14). After further washing, Chemiluminescent HRP Substrate; EMD Millipore) was used to visualize the protein bands. Finally, target protein levels 
A

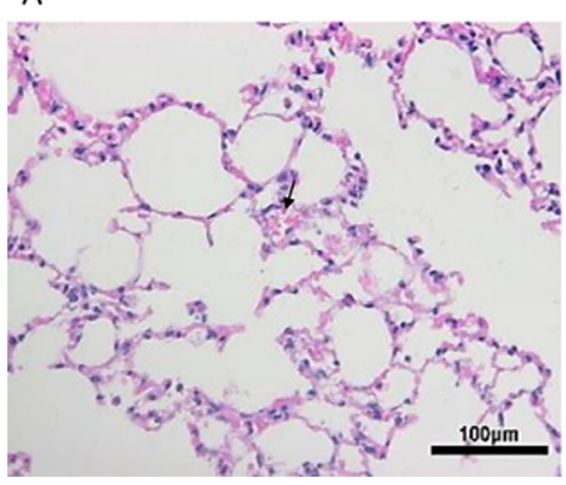

B

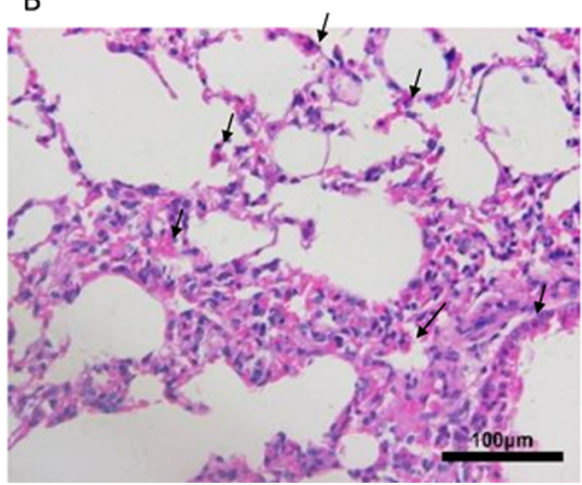

$\mathrm{C}$

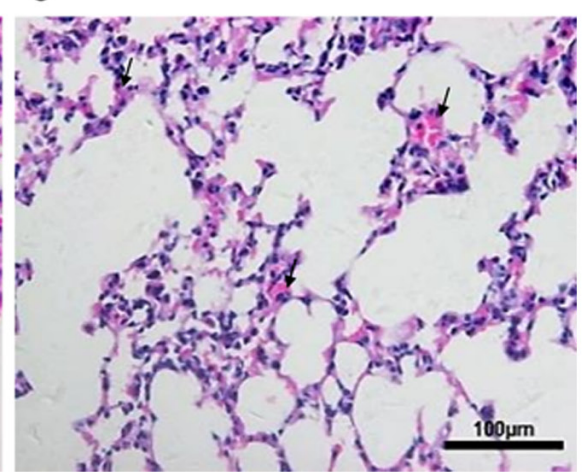

Figure 1. Lung tissue sections were stained with hematoxylin and eosin (magnification, x200). (A) Control group mice. (B) Mice in the CS group exhibited emphysematous changes. (C) Emphysema was partially ameliorated in the CS + cyclopamine group. Arrows signifify neurophils. CS, cigarette smoke.
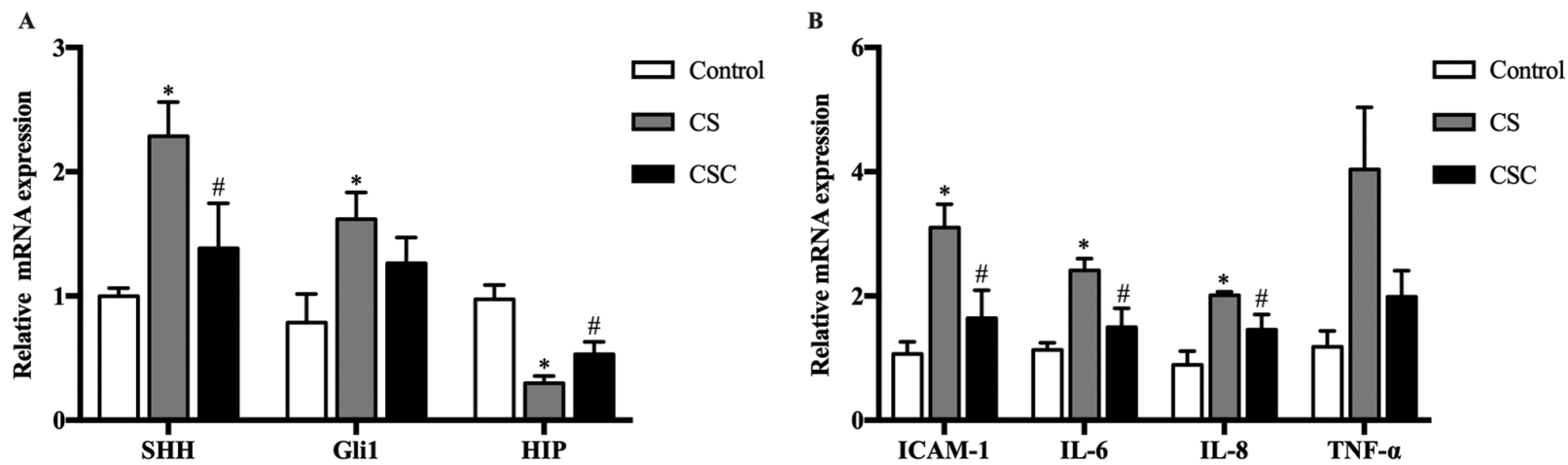

Figure 2. SHH, Gli1, HIP and inflammatory mediator mRNA expression in the three groups. (A) SHH, Gli1 and HIP mRNA expression levels. (B) ICAM-1, IL-6, IL-8 and TNF- $\alpha$ mRNA expression levels. ${ }^{*} \mathrm{P}<0.05$ vs. control; ${ }^{*} \mathrm{P}<0.05$ vs. CS. CS, cigarette smoke; CSC, CS + cyclopamine; Gli1, glioma-associated oncogene homolog 1; HIP, hedgehog-interacting protein; ICAM-1, intracellular adhesion molecule-1; SHH, sonic hedgehog.

relative to the internal control were calculated using Image $\mathrm{J}$ $1.52 \mathrm{v}$ software (National Institutes of Health).

Statistical analysis. Data were analyzed using SPSS (version 20.0; IBM Corp.) and were presented as the mean \pm SD. One-way ANOVA was performed to evaluate the differences between groups, followed by Scheffe's post hoc test for multiple comparisons. $\mathrm{P}<0.05$ was considered to indicate a statistically significant difference. The experiments were performed in triplicate.

\section{Results}

Lung histology and measurement of emphysema. Following exposure to CS for 24 weeks, the lungs of mice were histologically analyzed (Fig. 1). Compared with the control group, mice in the CS group exhibited focal enlargement of the alveoli and inflammatory cell infiltration, exhibiting neutrophil, lymphocyte and macrophage accumulation in lung parenchyma and interstitial spaces, with thickening of certain septa. By contrast, emphysema was partially ameliorated, as demonstrated by decreased inflammatory cell infiltration, in the lung tissues of CSC mice.

mRNA expression levels of SHH, Gli1, HIP and inflammatory mediators in the three groups. The mRNA expression levels of
SHH, Gli1 and HIP in the lung tissue of mice were compared among the three groups. The results revealed that SHH and Gli1 levels were significantly increased in the CS group compared with the control group (both $\mathrm{P}<0.05$ ), whereas their expression levels in the CSC group was lower than the CS group, significantly for SHH. HIP levels in the CS group were significantly lower compared with the control, but their levels in the CSC group were significantly higher than that in the in the CS $(\mathrm{P}<0.05)$ (Fig. 2A).

The mRNA expression levels of inflammatory mediators ICAM-1, IL-6 and IL-8 were significantly increased in the CS group compared with the control group $(\mathrm{P}<0.05 ;$ Fig. $2 \mathrm{~B})$, and these levels were significantly decreased following cyclopamine treatment, but not for TNF- $\alpha$.

Protein levels of SHH, Gli1, HIP and inflammatory mediators in the three groups. The protein expression levels of SHH, Gli1 and HIP in lung tissue were compared among the three groups. SHH and Gli1 levels in the CS group were significantly increased compared with the control group (both $\mathrm{P}<0.05$; Fig. 3). Furthermore, these expression levels were significantly decreased in the CSC group compared with the CS group (both $\mathrm{P}<0.05$ ). HIP levels were significantly decreased in the CS group compared with the control group, but significantly increased in the CSC group compared with the CS group (both $\mathrm{P}<0.05$; Fig. 3). 

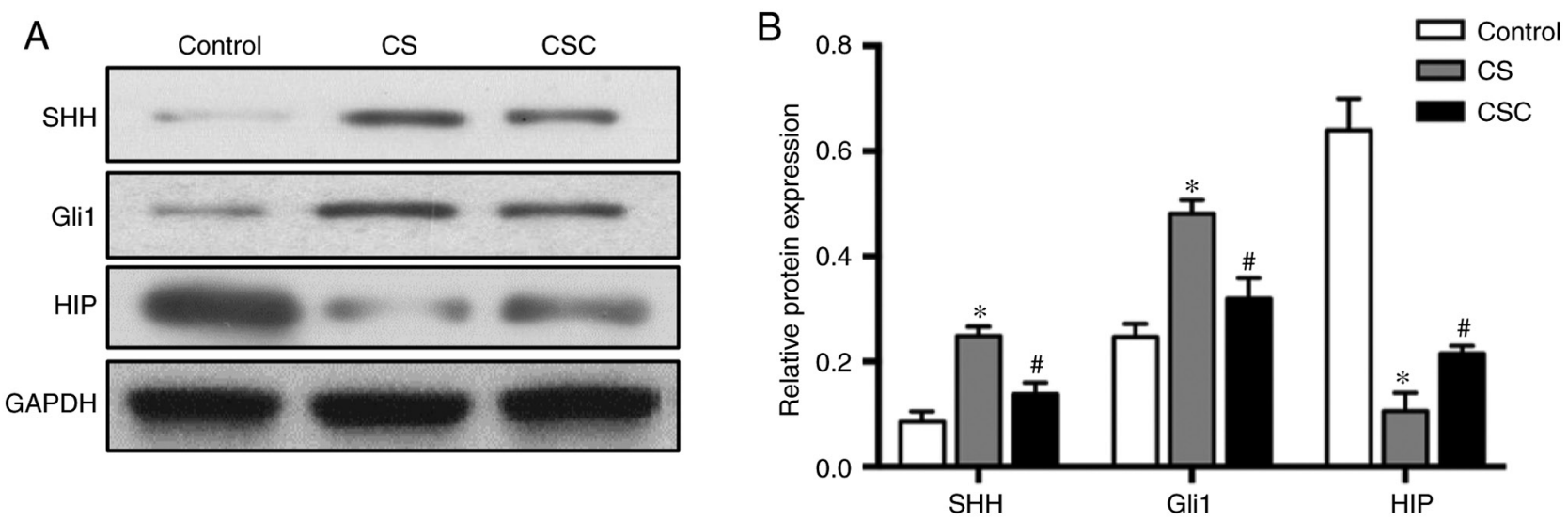

Figure 3. Western blotting results of SHH, Gli1 and HIP in the three groups. (A) SHH, Gli1 and HIP protein expression and (B) subsequent quantification. "P<0.05 vs. control; ${ }^{*} \mathrm{P}<0.05$ vs. CS. CS, cigarette smoke; CSC, cigarette smoke + cyclopamine; Gli1, glioma-associated oncogene homolog 1 ; HIP, hedgehog-interacting protein; SHH, sonic hedgehog.
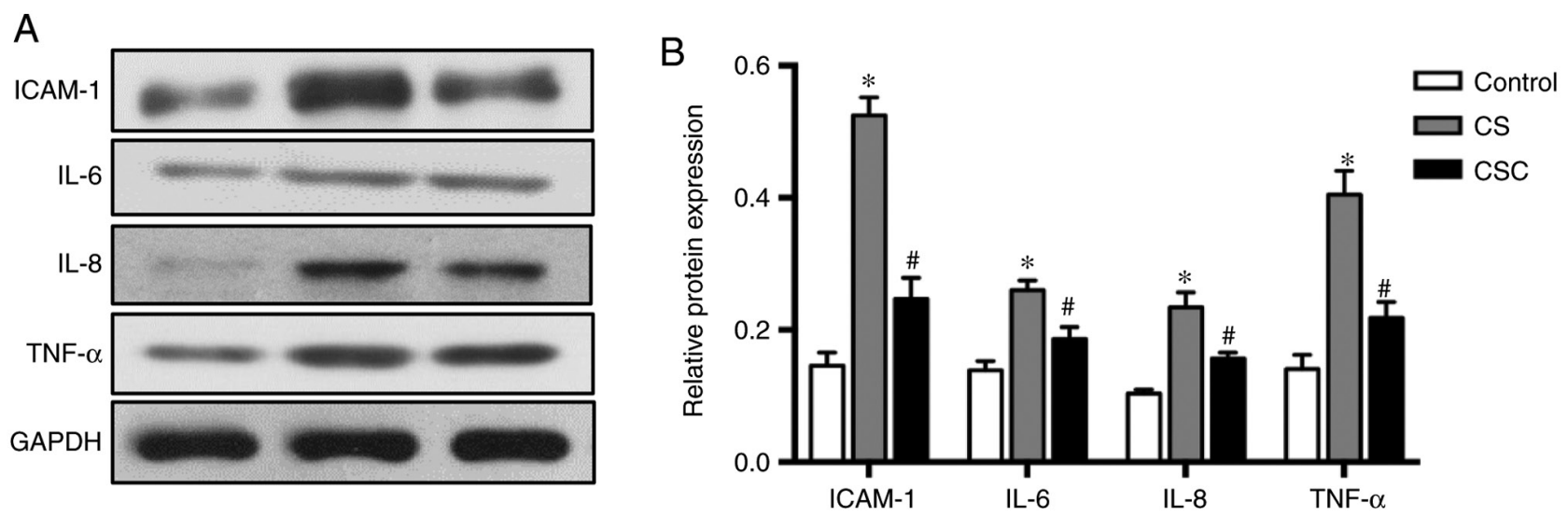

Figure 4. Western blotting of inflammatory mediators in the three groups. (A) ICAM-1, IL-6, IL-8 and TNF- $\alpha$ protein expression and (B) subsequent quantification. " $\mathrm{P}<0.05$ vs. control; ${ }^{\prime} \mathrm{P}<0.05$ vs. CS. CS, cigarette smoke; CSC, cigarette smoke + cyclopamine; ICAM-1, intracellular adhesion molecule-1.

The results of inflammatory mediators ICAM-1, IL-6, IL-8 and TNF- $\alpha$ followed similar trends. Protein expression levels were higher in the CS group compared with the control, but significantly lower following cyclopamine treatment (all $\mathrm{P}<0.05$; Fig. 4).

\section{Discussion}

The Hh signaling pathway serves a critical role in development, cell fate decisions and tissue growth (3). In adult tissues, the activation of the Hh pathway maintains homeostasis (15), which is typically regarded as a mitogenic cue during tissue development and injury or repair (16). A previous study demonstrated that Hh interacts with certain factors, such as MMPs, demonstrating an association with chronic lung inflammation (17). In the current study, the underlying mechanisms of Hh signaling were investigated in CS-induced emphysema and airway inflammation using an experimental animal model.

The mRNA and protein expression levels of SHH and Gli1 were significantly increased in the CS group when compared with the control, suggesting that CS stimulation aberrantly activated the Hh pathway, which is in line with a previous study (5). As a result of lung histopathological examination, the mice of the CS group presented with an emphysematous phenotype when compared with the controls, an observation that was partially ameliorated in the CSC group. This indicated that Hh signaling may have an important effect on cigarette-induced chronic airway disease. Therefore, the Hh signaling pathway must be strictly controlled to prevent inappropriate re-activation, which could be involved in the initiation and progression of inflammation and emphysema. Wang et al (14) revealed that the expansion of Hh activation in the alveolar mesenchyme may result in typical emphysema owing to alveolar damage and airspace enlargement (14). This suggested that the restriction of Hh activation in the alveolar mesenchyme is important for maintaining alveolar regeneration, and that emphysema can be caused by hyperactivation of the Hh pathway.

HIP, which is a negative regulator of $\mathrm{Hh}$, competitively binds to all three Hh ligands: $\mathrm{SHH}, \mathrm{IHH}$ and DHH (3). In the current study, HIP levels were decreased when mice were exposed to CS, suggesting that HIP may exert a protective role in COPD pathogenesis. These results were congruent to that of a previous study (7). Mice with HIP haplo-insufficiency $\left(\mathrm{HIP}^{+/-}\right)$are susceptible to severe airspace enlargement and emphysema with increasing lymphoid aggregates, enhancing 
the CS-induced lymphocyte activation pathways in the lungs (8). During early lung development, loss of HIP increases Hh activation, leading to defective branching morphogenesis (18). In adults, HIP expression persists in the lung tissue for the maintenance of lung homeostasis (7). HIP may regulate the function of the alveolar mesenchyme by restricting $\mathrm{Hh}$ signaling. Genome-wide association studies have identified certain loci, including the HIP gene, that is associated with COPD susceptibility $(19,20)$. Our previous study also identified various single nucleotide polymorphisms on the HIP gene in the Chinese Han population, which were associated with the susceptibility of COPD (21).

The effect of the Hh pathway in cigarette-induced airway inflammatory disease is not well established. Certain pro-inflammatory cytokines, such as IL-6, IL-8 and TNF- $\alpha$, which act as biomarkers of airway inflammation, have been involved in systemic oxidative inflammation, promoting COPD development (2). Hence, the mRNA and protein expression of various airway inflammatory mediators in the lung tissues of mice in the CS or CSC groups, including ICAM-1, IL-6, IL-8 and TNF- $\alpha$, were was measured in the current study. The results revealed that inflammation mediator expression levels were significantly increased in the CS group compared with the control group, but decreased following cyclopamine treatment. Therefore, aberrant activation of the Hh signaling pathway may partly enhance airway inflammation, whereas airway inflammatory reactions might be downregulated through decreased cytokine levels when $\mathrm{Hh}$ signaling is blocked. These pro-inflammatory molecules have also been reported to be regulated by $\mathrm{Hh}$ in other inflammatory diseases (22). Additional studies have demonstrated that the Hh pathway participates in inflammatory processes, not only in lung diseases, but also in other organ diseases. For example, the cyclopamin-induced inhibition of Hh signaling in rats with arthritis led to decreased TNF- $\alpha$, IL-1 $\beta$ and IL-6 levels (22). In gastritis, Gli1 upregulated IL-6, IL- $1 \beta$ and TNF- $\alpha$, and was regarded as the main promoter of inflammatory response (23). As such, previous studies have demonstrated that $\mathrm{Hh}$ signaling is capable of promoting the inflammatory process in the microenvironment (23). The results of the present study revealed that the protein level of TNF- $\alpha$ in the CS group was significantly higher compared with the control; however, while an increase was also observed in mRNA levels, this effect was not significant. Further studies should be performed to confirm the repeatability of the results obtained for this inflammatory mediator.

Hh signaling may regulate hepatocyte growth factor (HGF), which is a target gene of Hh in COPD. HGF is secreted by macrophage and mesenchyme cells in the airway, and it has been demonstrated to serve a crucial role in emphysema. Deficient HGF expression leads to emphysematous alveolar destruction, enlarged air spaces and chronic airway inflammation (24). However, further studies are required to clarify the potential pathways by which Hh signaling regulates inflammatory cytokines in COPD.

Notably, our previous study (9) indicated that Hh served a role in cigarette-induced airway inflammation through the regulation of certain inflammatory cytokines. Using the A549 cell model, we demonstrated the role of $\mathrm{Hh}$ in regulating some inflammatory mediators, but not emphysema (histological analysis of lung tissues). However, in the current study, another experiment was perforned using an animal model, with a focus on lung histology and measurement of emphysema, in addition to some inflammatory mediators, with the aim of clarifying the effect of Hh signaling on emphysema.

The current study had certain limitations. Additional evidence including cell or animal experiments is required to clarify the underlying mechanism with regard to the impact of the Hh pathway on COPD development. Furthermore, the current study focused on smoking-related airway inflammation, but in certain patients with COPD and no history of smoking, emphysema and airway inflammation would not be associated with cigarette smoke. Thus, further research is warranted to determine whether Hh signaling also participates in COPD development in these patients.

The present study revealed that Hh signaling might serve an important role in cigarette-induced emphysema and airway inflammation through the regulation of inflammatory cytokines. Therefore, diminishing the activation of Hh signaling may serve as a novel therapeutic strategy for patients with smoking-related COPD.

\section{Acknowledgements}

Not applicable.

\section{Funding}

No funding was received.

\section{Availability of data and materials}

The datasets used and/or analyzed during the current study are available from the corresponding author on reasonable request.

\section{Authors' contributions}

FYL and RC performed experiments, analyzed the data and wrote the manuscript. YG and MZ proposed the hypothesis, revised the manuscript and approved the version to be submitted. YG and MZ confirmed the authenticity of all the raw data. All authors have read and approved the final manuscript.

\section{Ethics approval and consent to participate}

All animal experiments were approved by the Institutional Animal Care and Use Committee of Shanghai Rui Jin Hospital, Shanghai Jiao Tong University (Shanghai, China; approval no. 105).

\section{Patient consent for publication}

Not applicable.

\section{Competing interests}

The authors declare that they have no competing interests. 


\section{References}

1. McCloskey SC, Patel BD, Hinchliffe SJ, Reid ED, Wareham NJ and Lomas DA: Siblings of patients with severe chronic obstructive pulmonary disease have significant risk of airflow obstruction. Am J Respir Crit Care Med 164: 1419-1424, 2001.

2. Bames PJ, Burney PG, Silverman EK, Celli BR, Vestbo J, Wedzicha JA and Wouters EFM: Chronic obstructive pulmonary disease. Nat Rev Dis Primers 1: 15076, 2015.

3. Briscoe $\mathrm{J}$ and Therond PP: The mechanisms of Hedgehog signalling and its roles in development and disease. Nat Rev Mol Cel Biol 14: 416-429, 2013.

4. Kasper M, Regl G, Frischauf AM and Aberger F: GLI1 transcription factors: Mediators of oncogenic Hedgehog signaling. Eur J Cancer 42: 437-445, 2006.

5. Lemjabbar-Alaoui H, Dasari V, Sidhu SS, Mengistab A, Finkbeiner W, Gallup M and Basbaum C: Wnt and Hedgehog are critical mediators of cigarette smoke-induced lung cancer. PLoS One 1: e93, 2006.

6. Kugler MC, Loomis CA, Zhao Z, Cushman JC, Liu L and Munger JS: Sonic Hedgehog signaling regulates myofibroblast function during alveolar septum formation in murine postnatal lung. Am J Respir Cell Mol Biol 57: 280-293, 2017.

7. Lao T, Jiang Z, Yun J, Qiu W, Guo F, Huang C, Mancini JD, Gupta K, Laucho-Contreras ME, Naing ZZC, et al: Hhip haploin sufficiency sensitizes mice to age-related emphysema. Proc Nat Acad Sci USA 113: E4681-E4687, 2016.

8. Lao T, Glass K, Qiu W, Polverino F, Gupta K, Morrow J, Dominic Mancini J, Vuong L, Perrella MA, Hersh CP, et al: Haploinsufficiency of Hedgehog interacting protein causes increased emphysema induced by cigarette smoke through network rewiring. Genome Med 7: 12, 2015.

9. Guo Y, Shi GC, Wan HY and Zhou M: Hedgehog signaling regulates the expression levels of inflammatory mediators in cigarette-induced airway inflammation. Mol Med Rep 17: 8557-8563, 2018.

10. Wu F, He ZQ, Ding R, Huang ZG, Jiang QX, Cui HM, Lin Y, Huang SB, Dai XL, Zhang JY, et al: Danhong promotes angiogenesis in diabetic mice after critical limb ischemia by activation of CSE-H2S-VEGF axis. Evid Based Complement Alternat Med 2015: 276263, 2015.

11. D'hulst AI, Vermaelen KY, Brusselle GG, Joos GF and Pauwels RA: Time course of cigarette smoke-induced pulmonary inflammation in mice. Eur Respir J 26: 204-213, 2005.

12. Chen X, Jin YT, Hou XM, Liu FQ and Wang YL: Sonic Hedgehog signaling: Evidence for its protective role protective role in endotoxin induced acute lung injury in mouse model. PLoS One 10: e0140886, 2015.

13. Livak KJ and Schmittgen TD: Analysis of relative gene expression data using real-time quantitative PCR and the 2(-Delta Delta $\mathrm{C}(\mathrm{T})$ ) method. Methods 25: 402-408, 2001.
14. Wang C, de Mochel NSR, Christenson SA, Cassandras M, Moon R, Brumwell AN, Byrnes L, Li A, Yokosaki Y, Shan P, et al: Expansion of Hedgehog disrupts mesenchymal identity and induces emphysema phenotype. J Clin Invest 128: 4343-4358, 2018.

15. Solanas G and Benitah SA: Regenerating the skin: A task for the heterogeneous stem cell pool and surrounding niche. Nat Rev Mol Cell Biol 14: 737-748, 2013.

16. Varjosalo $M$ and Taipale J: Hedgehog: Functions and mechanisms. Genes Dev 22: 2454-2472, 2008

17. Onishi H, Morisaki T, Nakao F, Odate S, Morisaki T and Katano M: Protein-bound polysaccharide decreases invasiveness and proliferation in pancreatic cancer by inhibition of Hedgehog signaling and HIF-1 $\alpha$ pathways under hypoxia. Cancer Lett 335: 289-298, 2013.

18. Chuang PT, Kawcak T and McMahon AP: Feedback control of mammalian Hedgehog signaling by the Hedgehog-binding protein, Hip1, modulates Fgf signaling during branching morphogenesis of the lung. Genes Dev 17: 342-347, 2003.

19. Hancock DB, Eijgelsheim M, Wilk JB, Gharib SA, Loehr LR, Marciante KD, Franceschini N, van Durme YMTA, Chen TH, Barr RG, et al: Meta-analyses of genome-wide association studies identify multiple loci associated with pulmonary function. Nat Genet 42: 45-52, 2010.

20. Pillai SG, Ge DL, Zhu GH, Kong XY, Shianna KV, Need AC, Feng S, Hersh CP, Bakke P, Gulsvik A, et al: A genome-wide association study in chronic obstructive pulmonary disease (COPD): Identification of two major susceptibility loci. PLoS Genet 5: e1000421, 2009.

21. Guo Y, Gong Y, Pan C, Qian Y, Shi G, Cheng Q, Li QY, Ren L, Weng QL, Chen Y, et al: Association of genetic polymorphisms with chronic obstructive pulmonary disease in the chinese han population: A case-control study. BMC Med Genomics 5: 64, 2012.

22. Li R, Cai L, Ding J, Hu CM, Wu TN and Hu XY: Inhibition of Hedgehog signal pathway by cyclopamine attenuates inflammation and articular cartilage damage in rats with adjuvant-induced arthritis. J Pharm Pharmacol 67: 963-971, 2015.

23. El-Zaatari M, Kao JY, Tessier A, Bai L, Hayes MM, Fontaine C, Eaton KA and Merchant JL: Gli1 deletion prevents helicobacter-induced gastric metaplasia and expansion of myeloid cell subsets. PLoS One 8: e58935, 2013.

24. Wang CQ, Cassandras M and Peng T: The role of Hedgehog signaling in adult lung regeneration and maintenance. J Dev Biol 7: 14, 2019.

This work is licensed under a Creative Commons Attribution-NonCommercial-NoDerivatives 4.0 International (CC BY-NC-ND 4.0) License. 